International Journal of Pure and Applied Mathematics

Volume 103 No. 1 2015, 133-153

ISSN: 1311-8080 (printed version); ISSN: 1314-3395 (on-line version)

url: http://www.ijpam.eu

doi: http://dx.doi.org/10.12732/ijpam.v103i1.11

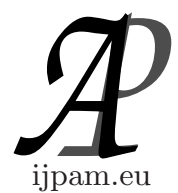

\title{
AN INTERVAL OF NO-ARBITRAGE PRICES FOR AMERICAN CONTINGENT CLAIMS IN INCOMPLETE MARKETS
}

\author{
Luca Di Persio $^{1 \S}$, Immacolata Oliva ${ }^{2}$ \\ ${ }^{1,2}$ Department of Computer Sciences \\ University of Verona \\ Strada le Grazie 15, 37134, Verona, ITALY
}

\begin{abstract}
In this paper we establish an arbitrage-free prices interval for American contingent claims in incomplete financial markets. Such an incompleteness derives from considering uncertain volatility. We use the notion of $G$-expectation, under which the corresponding canonical path is a $G$-Brownian Motion, and the related Itô stochastic calculus on suitable stopping time intervals, in a standard financial market characterized by a risk-less asset and one risky stock.
\end{abstract}

AMS Subject Classification: 60G40, 60G44, 91G20, 91G80, 91B25

Key Words: American contingent claim, $G$-expectation, pricing, uncertain volatility

\section{Introduction}

This paper aims at pricing and hedging American contingent claims under uncertain volatility models (UVM, from now on), in a financial market consisting in a risk-less asset and a risky stock price.

Received: May 26, 2015

(C) 2015 Academic Publications, Ltd. url: www.acadpubl.eu

$\S$ Correspondence author 
We assume that the underlying price process $S_{t}$ evolves according to the Black-Scholes model, which means that $S_{t}$ is an Itô process of the form $S_{t}=$ $r S_{t} \mathrm{~d} t+\sigma_{t} S_{t} \mathrm{~d} W_{t}$, where the drift coincides with the risk-free interest rate $r$, supposed to be constant, $\left(W_{t}\right)_{t \geq 0}$ is the classical Brownian Motion and the volatility parameter $\sigma_{t}$ is unknown and belongs to finite interval of values, namely $\sigma \in[\underline{\sigma}, \bar{\sigma}]$, with $0<\underline{\sigma}<\bar{\sigma}$.

The volatility lower, resp. upper, bound, i.e. $\underline{\sigma}$, resp. $\bar{\sigma}$, can be considered as input data of the problem and they are chosen according to the users' expectation as well as to the uncertainty of future prices fluctuations. As an example, it is rather standard to take such bounds as the extreme values or peaks of (historical) implied volatilities, see, e.g., [2].

One of the main reason behind the decision to consider UVM lies in the need to overcome the drawbacks of Black-Scholes model and, in particular, the assumption that the volatility surface, computed with respect to the variations of strike prices and expiration dates, has to be flat.

On the other hand, even if stochastic-volatility are able to better capture market empirical features, they typically implies the lack of market completeness, therefore perfect hedging is generally impossible.

By following the way paved in [18] in the case of European contingent claims, we obtain a closed interval for American contingent claim prices, within which no arbitrage opportunity may occur.

In order to take into account model uncertainty characterizing our model, we make use of the so-called $G$-expectations, with corresponding canonical path indicated by $G$-Brownian Motion, that was first introduced by Peng [10] and, then, developed by several authors, see, e.g., [6, 9, 16, 17]. We would like to recall that $G$-expectations are in particular sublinear expectations, and that such a family of expectations has gained a preeminent role in risk analysis. Indeed, the notion of sublinear expectation, and, more generally, of nonlinear expectation, is strictly connected with the concept, proposed at the end of the nineties in [1], of coherent risk measure. From that moment on, such a nonlinear approach was extensively applied in studying dynamic risk measures, see, e.g., [12] and [15], and to develop super-hedging and super-pricing approaches as, e.g., in $[4,5]$.

The decision to favor sublinear expectations, rather than more general nonlinear $g$-expectations, although for the latter a rich literature was already available, can be found in $[11,13]$, where Peng explains that the $G$-framework is not based on a classical probability space given a priori, while, in the $g$-framework, one needs to consider a probability measure, among an uncountable number of unknown ones essentially singular from each other, which has to be absolutely 
continuous with respect to a probability measure, typically a Wiener measure.

We want to recall that an American option with maturity $T$ may be exercised at any time before the option expires. By virtue of this and in order to define the corresponding payoff, it is essential to introduce the concept of stopping time $\tau$. Roughly speaking, this means that an event occurs at random time $\tau$ if, at any time $0 \leq t$ one can decide if the event has already occurred and then $\tau \leq t$, or is yet to occur, hence $\tau>t$, based on the information given by the filtration, up to time $t$. In such a context, the $G$-framework, as it was defined in [10], can not be used, as it provides the quasi-continuity of random variables involved. Nevertheless, in [9] latter drawback has been solved introducing an Itô stochastic calculus on stopping time intervals which allows to handle Itô integrals of the form $\int_{0}^{t \wedge \tau} \eta_{s} \mathrm{~d} B_{s}$, where $B_{s}$ is the $G$-Brownian Motion previously cited. In particular, such a result allows to treat the Itô integral for stochastic processes without assuming quasi-continuity and to deal with Itô formula for a general $C^{1,2}$-function.

The paper is organized as follows: in Section 2 we summarize the main definitions and properties related to the $G$-expectation framework, as well as the Itô stochastic calculus with respect to a stopping time interval, while in Section 3 the hedging problem for American contingent claims under uncertain volatility is formulated and results about the arbitrage-free interval are provided.

\section{2. $G$-expectations}

\subsection{Sublinear expectation}

In what follows, we introduce the main definitions and results concerning $G$ expectation theory. We will essentially proceed according to the scheme used in $[10,14]$ and then taken up in [18].

Definition 1. Let $\Omega \neq \emptyset$ be a given set of scenarios and consider a linear space $\mathcal{H}$ of real functions defined on $\Omega$, such that $\mathcal{H}$ contains all constants $c$ and such that $X \in \mathcal{H}$ implies $|X| \in \mathcal{H}$.

A sublinear expectation $\widehat{\mathbb{E}}$ on $\mathcal{H}$ is a functional $\widehat{\mathbb{E}}: \mathcal{H} \rightarrow \mathbb{R}$ such that the following properties hold

Monotonicity: if $X \geq Y$, then $\widehat{\mathbb{E}}(X) \geq \widehat{\mathbb{E}}(Y)$

Constant preserving: $\widehat{\mathbb{E}}(c)=c, c \in \mathbb{R}$

Sub-additivity: $\widehat{\mathbb{E}}(X+Y) \leq \widehat{\mathbb{E}}(X)+\widehat{\mathbb{E}}(Y)$ 
Positive homogeneity: $\widehat{\mathbb{E}}(\lambda X)=\lambda \widehat{\mathbb{E}}(X)$, for $\lambda \geq 0$

The triple $(\Omega, \mathcal{H}, \widehat{\mathbb{E}})$ is a sublinear expectation space.

Remark 2. The last two properties are often referred to as the so-called sub-linearity property and imply the convexity property, namely

$$
\widehat{\mathbb{E}}(\lambda X+(1-\lambda) Y) \leq \widehat{\mathbb{E}}(X)+(1-\lambda) \widehat{\mathbb{E}}(Y), \text { for all } \lambda \in[0,1] .
$$

The second and the third property guarantee the cash translatability property which is expressed by

$$
\widehat{\mathbb{E}}(X+c)=\widehat{\mathbb{E}}(X)+c, \text { for any } c \in \mathbb{R} .
$$

The sublinear expectation space looks like a classical probability space, where the $\sigma$-algebra is replaced by the linear space $\mathcal{H}$. Therefore, it makes sense to consider typical concepts of probability theory associated with linear expectations, such as the distribution of random variables, the independence between them and so on.

In order to do this, let us introduce the space of all bounded and Lipschitz continuous functions on $\mathbb{R}^{n}, n \geq 1$, denoted by $C_{b, \text { Lip }}\left(\mathbb{R}^{n}\right)$, for $n \geq 1$.

Remark 3. The space $C_{b, L i p}\left(\mathbb{R}^{n}\right)$ is introduced just for the sake of convenience, that is to say, it could be replaced by other spaces of functions, such as the space $\mathbb{L}^{\infty}\left(\mathbb{R}^{n}\right)$ of bounded Borel-measurable functions, the space $C_{b}\left(\mathbb{R}^{n}\right)$ of bounded continuous functions, the space $C_{b}^{k}\left(\mathbb{R}^{n}\right)$ of bounded and $k$ times differentiable functions, with bounded derivatives of all order less or equal to $k$, the space $C_{\text {unif }}\left(\mathbb{R}^{n}\right)$ of bounded and uniformly continuous functions, the space $C_{l, L i p}\left(\mathbb{R}^{n}\right)$ of locally Lipschitz functions or the space $L^{0}\left(\mathbb{R}^{n}\right)$ of Borel measurable functions, see [10] for further details.

Definition 4. Let $X=\left(X_{1}, \ldots, X_{n}\right)$ be a given $n$-dimensional random vector on $(\Omega, \mathcal{H}, \widehat{\mathbb{E}})$ and consider the functional $\widehat{\mathbb{F}}_{X}[\cdot]: C_{b, \text { Lip }}\left(\mathbb{R}^{n}\right) \rightarrow \mathbb{R}$ such that

$$
\varphi \in C_{b, \text { Lip }}\left(\mathbb{R}^{n}\right) \longmapsto \widehat{\mathbb{F}}_{X}[\varphi]:=\widehat{\mathbb{E}}[\varphi(X)] .
$$

Then, the triple $\left(\mathbb{R}, C_{b, L i p}\left(\mathbb{R}^{n}\right), \widehat{\mathbb{F}}_{X}[\cdot]\right)$ forms a sublinear expectation space and $\widehat{\mathbb{F}}_{X}$ is the distribution of $X$.

If the distribution $\widehat{\mathbb{F}}_{X}$ of $X \in \mathcal{H}$ is not a linear expectation, then we say that $X$ has a distributional uncertainty. Such a distribution is characterized by 
four parameters, namely

$$
\bar{\mu}:=\widehat{\mathbb{E}}[X], \underline{\mu}:=-\widehat{\mathbb{E}}[-X], \bar{\sigma}^{2}:=\widehat{\mathbb{E}}\left[X^{2}\right] ; \underline{\sigma}^{2}:=-\widehat{\mathbb{E}}\left[-X^{2}\right] .
$$

In particular, $\bar{\mu}$ and $\underline{\mu}$ represent the mean-uncertainty of $X$, while $\bar{\sigma}^{2}$ and $\sigma^{2}$ describe the variance-uncertainty of $X$. In this paper, we focus on varianceuncertainty, hence we set $\bar{\mu}=\underline{\mu}$.

Definition 5. Let $X_{1}$ and $X_{2}$ be two random vectors, defined respectively in the sublinear expectation spaces $\left(\Omega_{1}, \mathcal{H}_{1}, \widehat{\mathbb{E}}_{1}\right)$ and $\left(\Omega_{2}, \mathcal{H}_{2}, \widehat{\mathbb{E}}_{2}\right)$. Such random vectors are said to be identically distributed, in symbols $X_{1} \sim X_{2}$, if

$$
\widehat{\mathbb{E}}_{1}\left[\varphi\left(X_{1}\right)\right]=\widehat{\mathbb{E}}_{2}\left[\varphi\left(X_{2}\right)\right] \text {, for all } \varphi \in C_{b, L i p}\left(\mathbb{R}^{n}\right) .
$$

The independence of random variables under a sublinear expectation space is a very interesting issue.

Definition 6. In a sublinear expectation space $(\Omega, \mathcal{H}, \widehat{\mathbb{E}})$, a random vector $Y=\left(Y_{1}, \ldots, Y_{n}\right) \in \mathcal{H}$ is said to be independent to another random vector $X=\left(X_{1}, \ldots, X_{m}\right) \in \mathcal{H}$ under $\widehat{\mathbb{E}}$ if, for each test function $\varphi \in C_{b, \text { Lip }}\left(\mathbb{R}^{m} \times \mathbb{R}^{n}\right)$, we have

$$
\widehat{\mathbb{E}}[\varphi(X, Y)]=\widehat{\mathbb{E}}\left[\widehat{\mathbb{E}}[\varphi(x, Y)]_{x=X}\right] .
$$

It is important to notice that, unlike what occurs in the linear case, under the sublinear expectation if $X$ is independent to $Y$, this does not imply that $Y$ is independent to $X$, too. Hence, we define $\bar{X}$ an independent copy of $X$ if $X \sim \bar{X}$ and $\bar{X}$ is independent to $X$.

As well as in the classical probability theory, Gaussian distribution plays a central role also under a sublinear expectation. For the sake of simplicity, here we restrict ourselves to the one-dimensional case $n=1$, but the generalization to the $n$-dimensional, $n>1$, case is straightforward.

Definition 7. In a sublinear expectation space $(\Omega, \mathcal{H}, \widehat{\mathbb{E}})$ a random variable $X \in \mathcal{H}$ with $\bar{\sigma}^{2}=\widehat{\mathbb{E}}\left[X^{2}\right]$ and $\underline{\sigma}^{2}=-\widehat{\mathbb{E}}\left[-X^{2}\right]$, is said to be $G-$ normal distributed, denoted by $X \sim \mathcal{N}\left(0,\left[\bar{\sigma}^{2}, \underline{\sigma}^{2}\right]\right)$, if, for each independent copy $Y$ of $X$, we have

$$
a X+b Y \sim \sqrt{a^{2}+b^{2}} X, \forall a, b \geq 0 .
$$

We recall that a $G$-normal distributed random variable $X$ has no meanuncertainty, that is, $\widehat{\mathbb{E}}[X]=\widehat{\mathbb{E}}[-X]=0$, see $[10]$. 
Moreover, such a distribution on the sublinear expectation space is the uniquely defined sublinear distribution on $\left(\mathbb{R}, C_{b, L i p}(\mathbb{R})\right)$ and it can be obtained by solving a suitable parabolic partial differential equation (PDE), called the $G$-heat equation. More rigorously, one can prove that, if $X$ is a $G$-normal distributed random variable, then

$$
u(t, x):=\widehat{\mathbb{E}}[\varphi(x+\sqrt{t} X)],(t, x) \in[0, \infty) \times \mathbb{R}, \varphi \in C_{b, L i p}(\mathbb{R} \times \mathbb{R}),
$$

is the unique viscosity solution of

$$
\left\{\begin{array}{l}
\partial_{t} u-G\left(\partial_{x x}^{2} u\right)=0 \\
u(0, \cdot)=\varphi(\cdot)
\end{array},\right.
$$

see $[10]$ for details.

The function $G$ is the generating function of eq. (2), such that

$$
G(y):=\frac{1}{2} \bar{\sigma}^{2} y^{+}-\frac{1}{2} \underline{\sigma}^{2} y^{-}
$$

where $\bar{\sigma}^{2}=\widehat{\mathbb{E}}\left[X^{2}\right], \underline{\sigma}^{2}=-\widehat{\mathbb{E}}\left[-X^{2}\right], y^{+}:=\max (0, y)$ and $y^{-}:=\max (0,-y)$.

\subsection{G-Brownian Motion and $G$-expectations}

Let $(\Omega, \mathcal{H}, \widehat{\mathbb{E}})$ be a sublinear expectation space, then a stochastic process related to $(\Omega, \mathcal{H}, \widehat{\mathbb{E}})$, is nothing but a family of random variables $X_{t} \in \mathcal{H}, t \geq 0$.

Roughly speaking, a $G$-Brownian motion is a continuous stochastic process with independent and stationary increments under a given sublinear expectation, namely the following definition holds

Definition 8. A process $\left(B_{t}\right)_{t \geq 0}$ in a sublinear expectation space $(\Omega, \mathcal{H}, \widehat{\mathbb{E}})$ is called a $G$-Brownian motion if, for each $n \in \mathbb{N}, 0 \leq t_{1}, \ldots, t_{n}<\infty$ and $B_{t_{1}}, \ldots, B_{t_{n}} \in \mathcal{H}$, the following properties are satisfied:

(a) $B_{0}=0$

(b) for each $t, s \geq 0$, the increment $B_{t+s}-B_{t}$ is independent to $\left(B_{t_{1}}, \ldots, B_{t_{n}}\right)$ and $\mathcal{N}\left(0,\left[\underline{\sigma}^{2} s, \bar{\sigma}^{2} s\right]\right)$-distributed, for each $n \in \mathbb{N}$ and $0 \leq t_{1} \leq \ldots \leq t_{n} \leq$ $t$.

We note that the letter $G$ stands for the generating function characterizing the process, i.e.

$$
G(y):=\widehat{\mathbb{E}}\left[y B_{1}^{2}\right], y \in \mathbb{R}
$$


For each fixed time horizon $T \geq 0$, let us consider the space of real-valued continuous paths, starting at zero, denoted by $\Omega_{T}:=C_{0}([0, T], \mathbb{R})$, and define the space of finite dimensional cylinder random variables

$$
L_{i p}\left(\Omega_{T}\right):=\left\{\varphi\left(B_{t_{1}}, \ldots, B_{t_{n}}\right): t_{i} \in[0, T], \varphi \in C_{b, L i p}\left(\mathbb{R}^{n}\right)\right\} .
$$

We are going to show how a sublinear expectation on $\left(\Omega_{T}, L_{i p}\left(\Omega_{T}\right)\right)$ can be defined, in such a way that the corresponding canonical process is a $G$-Brownian Motion. Such a sublinear expectation is called $G$-expectation and it will be indicated by $\mathbb{E}_{G}$.

Let us consider a sublinear expectation space $(\widetilde{\Omega}, \widetilde{\mathcal{H}}, \widetilde{\mathbb{E}})$ and a sequence of $G$-normal distributed random variables $\xi_{i}$ such that $\xi_{i+1}$ is independent to $\left(\xi_{1}, \ldots, \xi_{i}\right)$, for $i \geq 1$.

In addition, let us define the canonical process $B_{t}(\omega):=\omega_{t}, t \in[0, T]$, for $\omega \in \Omega_{t}$ and set

$$
\begin{aligned}
X & :=\varphi\left(B_{t_{1}}-B_{t_{0}}, B_{t_{2}}-B_{t_{1}}, \ldots, B_{t_{n}}-B_{t_{n-1}}\right) \in L_{i p}\left(\Omega_{T}\right) \\
\mathbb{E}_{G}[X] & :=\widetilde{\mathbb{E}}\left[\varphi\left(\sqrt{t_{1}-t_{0}} \xi_{1}, \ldots, \sqrt{t_{n}-t_{n-1}} \xi_{n}\right)\right],
\end{aligned}
$$

for some $\varphi \in C_{b, \operatorname{Lip}}\left(\mathbb{R}^{n}\right), 0 \leq t_{1} \leq \ldots \leq t_{n} \leq T$.

It follows that the functional $\mathbb{E}_{G}$ consistently defines a sublinear expectation on $L_{i p}\left(\Omega_{T}\right)$. Such a sublinear expectation is called $G$-expectation and the canonical process $\left(B_{t}\right)_{t \geq 0}$ on the sublinear expectation space $\left(\Omega_{T}, L_{i p}\left(\Omega_{T}\right), \mathbb{E}_{G}\right)$ is called a $G$-Brownian Motion.

As it is well explained in $[14$, Ch.1, Sec.4], every sublinear expectation $\widehat{\mathbb{E}}$ can be continuously extended to a Banach space $\left(\widehat{\mathcal{H}}_{p},\|\cdot\|_{p}\right)$, for each fixed $p \geq 1$, where it continues to be a sublinear expectation, with $\widehat{\mathcal{H}}_{p}$ is the completion of $\mathcal{H} / \mathcal{H}_{0}^{p}, \mathcal{H}_{0}^{p}:=\left\{X \in \mathcal{H}, \widehat{\mathbb{E}}\left[|X|^{p}=0\right]\right\}$ and $\|X\|_{p}:=\left(\widehat{\mathbb{E}}\left[|X|^{p}\right]\right)^{1 / p}$, for $X \in \mathcal{H}$.

For our purpose, we will consider the following spaces:

- $\Omega_{T}^{t}:=\left\{\omega \cdot \wedge t: \omega \in \Omega_{T}\right\}$, for each $t \in[0, T]$

- $B_{b}\left(\Omega_{T}\right)$ (resp. $\left.B_{b}\left(\Omega_{T}^{t}\right)\right)$ the space of all bounded $\mathcal{B}\left(\Omega_{T}\right)$-measurable real functions (resp. the space of all bounded $\mathcal{B}\left(\Omega_{T}^{t}\right)$-measurable real functions), where $\mathcal{B}\left(\Omega_{T}\right)$ is the Borel $\sigma$-algebra of $\Omega$ (resp. $\mathcal{B}\left(\Omega_{T}^{t}\right)$ is the Borel $\sigma$-algebra of $\Omega_{t}$ )

- $L_{G}^{p}\left(\Omega_{T}\right), p \geq 1$, the completion of $L_{i p}\left(\Omega_{T}\right)$, defined in eq. (4), under the norm $\|X\|_{p}:=\left(\mathbb{E}_{G}\left[|X|^{p}\right]\right)^{1 / p}$ 
- $L_{*}^{p}\left(\Omega_{T}\right), p \geq 1$, (resp. $L_{*}^{p}\left(\Omega_{T}^{t}\right), p \geq 1$ ) the completion of $B_{b}\left(\Omega_{T}\right)$ (resp. the completion of $\left.B_{b}\left(\Omega_{T}^{t}\right)\right)$ under the norm $\|X\|_{p}:=\left(\mathbb{E}_{G}\left[|X|^{p}\right]\right)^{1 / p}$

- $M_{*}^{p}(0, T), p \geq 1$, the completion of $M_{b, 0}(0, T)$ under the norm

$$
\|\eta\|_{M_{*}^{p}(0, T)}:=\left(\mathbb{E}_{G}\left[\int_{0}^{T}\left|\eta_{t}\right|^{p} \mathrm{~d} t\right]\right)^{1 / p},
$$

where $M_{b, 0}(0, T)$ represents the collection of simple processes

$$
\eta_{t}(\omega):=\sum_{i=0}^{N-1} \xi_{i}(\omega) \mathbb{1}_{\left[t_{i}, t_{i+1}\right)(t)}, \forall t \in[0, T],
$$

with $\left\{0, t_{1}, \ldots, t_{N-1}, T\right\}$ a partition of $[0, T]$ and $\xi_{i} \in B_{b}\left(\Omega_{T}^{t_{i}}\right)$, for $i=$ $1, \ldots, N-1$.

Remark 9. We want to underline that the spaces $M_{*}^{p}(0, T), p \geq 1$, turn to be useful in the construction of Itô integral with respect to a $G$-Brownian Motion , see, e.g., [14], as we will see later on. The stochastic processes $\eta_{t}(\omega):=$ $\sum_{i=0}^{N-1} \xi_{i}(\omega) \mathbb{1}_{\left[t_{i}, t_{i+1}\right)(t)}$, for all $t \in[0, T]$ and for all $\xi_{i} \in L_{*}^{p}\left(\Omega_{t_{i}}\right), i=1, \ldots, N-$ 1 , are also in $M_{*}^{p}(0, T)$.

Moreover, we have that

$$
M_{*}^{p}(0, T) \supset M_{*}^{q}(0, T), \quad L_{*}^{p}\left(\Omega_{T}\right) \supset L_{G}^{p}\left(\Omega_{T}\right),
$$

for all $1 \leq p \leq q$.

The following Proposition will be used in next Section and its proof can be found in [9, Sec.3].

Proposition 10. For each $p \geq 1$, let $X, \eta \in M_{*}^{p}(0, T)$, with $\eta$ bounded. Then, $X \eta \in M_{*}^{p}(0, T)$.

\subsection{Relationship between G-Brownian Motion and classical Brownian Motion}

Let us consider a given family of probability measures, say $\mathscr{P}$, on the measurable space $(\Omega, \mathcal{B}(\Omega))$, being $\mathcal{B}(\Omega)$ the Borel $\sigma$-algebra. A sublinear expectation $\widehat{\mathbb{E}}$ is the upper expectation of $\mathscr{P}$ if

$$
\widehat{\mathbb{E}}[X]=\sup _{P \in \mathscr{P}} \mathbb{E}_{P}[X], \text { for each } X \in C_{b, \text { Lip }}(\mathbb{R}) .
$$


In our context we are interested in choosing such a family of probability measures, with respect to which the $G$-expectation $\mathbb{E}_{G}$ results to be an upper expectation, therefore it is useful to formalize the link between the $G$-framework and the classical probability setting.

Let $\left(\Omega_{T}, \mathcal{B}\left(\Omega_{T}\right), P\right)$ be a probability space, with $\mathcal{B}\left(\Omega_{T}\right)$ the related Borel $\sigma$-algebra and consider a classical Brownian Motion $W=\left(W_{t}\right)_{t \geq 0}$ defined on it and such that it generates the filtration $\mathcal{F}_{t}=\sigma\left\{W_{s}, 0 \leq s \leq t\right\} \vee \mathcal{N}, \mathcal{N}$ being the collection of $P$-null subsets. For a fixed $t \geq 0$, we define $\mathcal{F}_{s}^{t}:=$ $\sigma\left\{W_{t+u}-W_{t}, 0 \leq u \leq s\right\} \vee \mathcal{N}$ together with $\mathbb{F}=\left(\mathcal{F}_{t}\right)_{t \geq 0}$ and $\mathbb{F}^{t}=\left(\mathcal{F}_{s}^{t}\right)_{s \geq 0}$.

Moreover, let $\Theta:=[\underline{\sigma}, \bar{\sigma}]$ and denote by $\mathcal{A}_{t, T}^{\Theta}$ the collection of all $\Theta$-valued $\mathbb{F}_{s}^{t}$-adapted process on an interval $[t, T]$. For each fixed $\theta \in \mathcal{A}_{t, T}^{\Theta}$, we define

$$
B_{T}^{t, \theta}:=\int_{t}^{T} \theta_{s} \mathrm{~d} W_{s}
$$

Finally, we set $\mathscr{P}_{1}:=\left\{P^{\theta}: \theta \in \mathcal{A}_{t, T}^{\Theta}\right\}$ and $\mathscr{P}:=\overline{\mathscr{P}_{1}}$ the closure of $\mathscr{P}_{1}$ under the topology of weak convergence. Hence, $\mathscr{P}_{1}$ is tight and $\mathscr{P}$ is weakly compact, as it is shown in [3, Prop. 50].

The following Proposition, proved in [3, Prop. 49], establishes that the $G$-expectation can be equivalently defined in terms of upper expectation

Proposition 11. For any $\varphi \in C_{b, L i p}\left(\mathbb{R}^{n}\right), n \in \mathbb{N}, 0=t_{0} \leq t_{1} \leq \ldots \leq$ $t_{n} \leq T$, we have

$$
\begin{aligned}
\mathbb{E}_{G}\left[\varphi\left(B_{t_{1}}-B_{t_{0}}, \ldots, B_{t_{n}}-B_{t_{n-1}}\right)\right] & \\
& =\sup _{\theta \in \mathcal{A}_{t, T}^{\ominus}} \mathbb{E}^{P}\left[\varphi\left(B_{t_{1}}^{0, \theta}, \ldots, B_{t_{n}}^{t_{n-1}, \theta}\right)\right] \\
& =\sup _{\theta \in \mathcal{A}_{t, T}^{\Theta}} \mathbb{E}^{P^{\theta}}\left[\varphi\left(B_{t_{1}}-B_{t_{0}}, \ldots, B_{t_{n}}-B_{t_{n-1}}\right)\right] \\
& =\sup _{P^{\theta} \in \mathscr{P}} \mathbb{E}^{P^{\theta}}\left[\varphi\left(B_{t_{1}}-B_{t_{0}}, \ldots, B_{t_{n}}-B_{t_{n-1}}\right)\right],
\end{aligned}
$$

where $P^{\theta}$ is the law of the process $B_{t}^{0, \theta}:=\int_{0}^{t} \theta_{s} \mathrm{~d} W_{s}, t \geq 0, \theta \in \mathcal{A}_{0, T}^{\Theta}$.

We recall the standard capacity-related vocabulary, which is widely used within the $G$-framework.

Definition 12. We say that a set $A$ is polar if $P(A)=0, \forall P \in \mathscr{P}$. A property holds quasi surely (q.s.) if it holds outside a polar set. 
Remark 13. Recall that a mapping $X: \Omega_{T} \rightarrow \mathbb{R}$ is said to be quasicontinuous (q.c.) if, for all $\epsilon>0$, there exists an open set $O$ with $\sup _{P \in \mathscr{P}} P(O)<$ $\epsilon$, such that $\left.X\right|_{O^{c}}$ is continuous. Moreover, $X: \Omega_{T} \rightarrow \mathbb{R}$ has a quasi-continuous version if there exists a quasi-continuous function $Y: \Omega_{T} \rightarrow \mathbb{R}$ with $X=Y$ q.s. Then,

$$
L_{G}^{p}\left(\Omega_{T}\right)=\left\{X \in L^{0}\left(\Omega_{T}\right): \lim _{n \rightarrow 0} \mathbb{E}_{G}\left[|X|^{p} \mathbb{1}_{\{|X| \geq n\}}\right]=0\right\},
$$

where $L^{0}\left(\Omega_{T}\right)$ denotes the space of all Borel-measurable real-valued functions on $\Omega_{T}$ and $X$ has a q.c. version.

An analogous result holds when we consider the space $L_{*}^{p}\left(\Omega_{T}\right)$ without requiring the quasi-continuity property, that is,

$$
L_{*}^{p}\left(\Omega_{T}\right)=\left\{X \in L^{0}\left(\Omega_{T}\right): \lim _{n \rightarrow 0} \mathbb{E}_{G}\left[|X|^{p} \mathbb{1}_{\{|X| \geq n\}}\right]=0\right\},
$$

where $L^{0}\left(\Omega_{T}\right)$ denotes the space of all Borel-measurable real-valued functions on $\Omega_{T}$.

\subsection{Stochastic calculus of Itô type}

In $[10,14]$, the stochastic integral with respect to the $G$-Brownian Motion $\left(B_{t}\right)_{t \geq 0}$ is introduced, and then generalized in order to take into consideration larger spaces of stochastic processes, see [9] for further details.

Definition 14. For any simple process $\eta \in M_{b, 0}(0, T)$, the stochastic integral with respect to the $G$-Brownian Motion $\left(B_{t}\right)_{t \geq 0}$ is

$$
I(\eta)=\int_{0}^{T} \eta_{s} \mathrm{~d} B_{s}:=\sum_{j=0}^{N-1} \xi_{j}\left(B_{t_{j-1}}-B_{t_{j}}\right) .
$$

The mapping $I: M_{b, 0}(0, T) \rightarrow L_{*}^{2}\left(\Omega_{T}\right)$ is a linear continuous mapping and thus can be continuously extended to $I: M_{*}^{2}(0, T) \rightarrow L_{*}^{2}\left(\Omega_{T}\right)$, with

$$
\begin{aligned}
\mathbb{E}_{G}\left[\int_{0}^{T} \eta_{s} \mathrm{~d} B_{s}\right] & =0 \\
\mathbb{E}_{G}\left[\left(\int_{0}^{T} \eta_{s} \mathrm{~d} B_{s}\right)^{2}\right] & \leq \bar{\sigma}^{2} \mathbb{E}_{G}\left[\int_{0}^{T} \eta_{s}^{2} \mathrm{~d} s\right]
\end{aligned}
$$


Further properties shared by Itô integral with respect to a $G$-Brownian Motion can be found in [9, Sec.3].

Due to its usefulness, we just report the following proposition, see [9, Prop. $3.7]$

Proposition 15. The Itô integral $\int_{0}^{t} \eta_{s} \mathrm{~d} B_{s}, \eta \in M_{*}^{2}(0, T)$, for $t \leq T$, results to be continuous in $t$ q.s. Furthermore, for every $\eta \in M_{*}^{2}(0, T)$, we have

$$
\int_{0}^{\cdot} \eta_{s} \mathrm{~d} B_{s} \in M_{*}^{2}(0, T)
$$

Because of its importance in what follows and since it characterizes the statistic uncertainty component of the $G$-Brownian Motion, we are going to introduce the so-called quadratic variation process of the $G$-Brownian Motion $\left(B_{t}\right)_{t \geq 0}$.

Definition 16. The quadratic variation process of $B_{t}$ is an increasing stochastic process such that

$$
\langle B\rangle_{t}=B_{t}^{2}-2 \int_{0}^{T} B_{s} \mathrm{~d} B_{s}, t \leq T, \text { with }\langle B\rangle_{0}=0 .
$$

A very interesting point of the quadratic variation process $\langle B\rangle$ is that

$$
\langle B\rangle_{t+s}-\langle B\rangle_{s}=\left\langle B_{t+s}-B_{s}\right\rangle_{t}, \text { for each } s \geq 0,
$$

therefore the quadratic variation process has independent increments and we also have that $\mathbb{E}_{G}\left[\langle B\rangle_{t}^{2}\right]=\sigma^{2} t^{2}$, hence the mean-uncertainty is concentrated in itself and this could be applied to measure the mean-uncertainty of risky positions.

It is possible to define a stochastic integral with respect to $\mathrm{d}\langle B\rangle_{t}$, by considering, for any stochastic process $\eta \in M_{*}^{1}(0, T)$,

$$
Q(\eta)=\int_{0}^{T} \eta_{s} \mathrm{~d}\langle B\rangle_{s}:=\sum_{j=0}^{N-1} \xi_{j}\left(\langle B\rangle_{t_{j-1}}-\langle B\rangle_{t_{j}}\right)
$$

and

$$
\mathbb{E}_{G}\left[\left(\int_{0}^{T} \eta_{s} \mathrm{~d} B_{s}\right)^{2}\right]=\mathbb{E}_{G}\left[\int_{0}^{T} \eta_{s}^{2} \mathrm{~d}\langle B\rangle_{s}\right], \forall \eta \in M_{*}^{2}(0, T) .
$$


Because of the financial applications of such mathematical framework, which will be examined in Section 3, it is necessary to introduce a stochastic calculus concerning stopping times, by following [9, Sec. 4].

Recall that a stopping time $\tau$ relative to the filtration $\mathbb{F}$ is a map on $\Omega_{T}$ with values in $[0, T]$ such that, for every $t \leq T,\{\tau \leq t\} \in \mathcal{F}_{t}$. It is possible to have a Itô's integral defined on $[0, \tau]$, where $\tau$ is a stopping time. In particular, for each stopping time $\tau$ and for each stochastic process $\eta \in M_{*}^{p}(0, T)$, we have

$$
\int_{0}^{t \wedge \tau} \eta_{s} \mathrm{~d} B_{s}=\int_{0}^{t} \mathbb{1}_{[0, \tau]}(s) \eta_{s} \mathrm{~d} B_{s} \quad \text { q.s. }
$$

Remark 17. Prop. (10) guarantees that, for each stopping time $\tau$, the stochastic process $\mathbb{1}_{[0, \tau]}(\cdot) \eta$ is an element of the space $M_{*}^{p}(0, T)$, for every $\eta \in$ $M_{*}^{p}(0, T)$.

\section{American contingent claims}

\subsection{The financial market}

The standard model for financial markets provides for $d+1$ continuously traded assets. One of these is the risk-less asset, the remaining $d$ are subjected to systematic risk.

For the sake of convenience, let us investigate a financial market $\mathcal{M}$, consisting in one risk-less asset, called bond or bank account, and one risky asset, called stock, whose dynamics evolve according to

$$
\left\{\begin{array}{ll}
\mathrm{d} \gamma_{t}=r \gamma_{t} \mathrm{~d} t, & \gamma_{0}=1 \\
\mathrm{~d} S_{t}=r S_{t} \mathrm{~d} t+S_{t} \mathrm{~d} B_{t}, & S_{0}=x_{0}
\end{array},\right.
$$

where $r$ is the interest rate, supposed to be a nonnegative integer constant, and $\left(B_{t}\right)_{t \geq 0}$ is the canonical $G$-Brownian Motion, introduced in Section 2, with parameters $\bar{\sigma}>0$ and $\underline{\sigma}>0$.

In particular, hereafter consider the filtered sublinear expectation space $\left(\Omega_{T}, \mathcal{B}\left(\Omega_{T}\right), \mathbb{F}, \mathscr{P}\right)$ together with the canonical $G$-Brownian Motion $B=\left(B_{t}\right)_{t \geq 0}$.

Recall that $\Omega_{T}:=C_{0}([0, T], \mathbb{R}), \mathcal{B}\left(\Omega_{T}\right)$ is the corresponding Borel $\sigma$-algebra, $\mathbb{F}:=\left(\mathcal{F}_{t}\right)_{t \geq 0}$ is the filtration generated by $B$ and $\mathscr{P}$ is the set of probability measures, with respect to which the $G$-expectation $\mathbb{E}_{G}$ is an upper expectation, as we have already pointed out in Sec. 2. 
Let us observe that the non-risky asset follows a deterministic dynamic, hence we are able to provide the corresponding price, by integrating the first equation in (17), so that $\gamma_{t}=e^{r t}$. We denote by $\gamma_{t}^{-1}$ the discount factor, that is, $\gamma_{t}^{-1}=e^{-r t}$.

We require $t \in[0, T]$, where $0<T<\infty$ is the time horizon, unless explicitly stated otherwise.

Definition 18. An $\mathbb{F}$-adapted valued process $\pi: L_{*}^{1}\left(\Omega_{T}\right) \rightarrow \mathbb{R}$ is called portfolio process.

A nonnegative $\mathbb{F}$-adapted process $C: L_{*}^{1}\left(\Omega_{T}\right) \rightarrow[0, \infty)$ with increasing, right-continuous paths and $C_{0}=0, C_{T}<\infty$ q.s. is called cumulative consumption process.

Definition 19. Given a capital $y$, a portfolio process $\pi$ and cumulative consumption $C$, the solution $X:=X^{y, \pi, C}$ of the following linear stochastic equation

$$
\mathrm{d} X_{t}=X_{t}\left(1-\pi_{t}\right) \frac{\mathrm{d} \gamma_{t}}{\gamma_{t}}+X_{t} \pi_{t} \frac{\mathrm{d} S_{t}}{S_{t}}-\mathrm{d} C_{t}, X_{0}=y
$$

defines the wealth process corresponding to the triple $(y, \pi, C)$.

From a financial point of view, $\pi_{t}$ represents the amount of the agent's wealth that is invested in the stock at time $t$, and this amount may be positive or negative, which means that short-selling of stocks is permitted, whereas $X_{t}-\pi_{t}$ is the amount not invested in stocks and put into the bank-account, whose negative values correspond to borrowing rather than saving, at the interest rate $r$.

Let us observe that eq. (18) can be written in the following slightly different form using eq. (17)

$$
\frac{\mathrm{d} \gamma_{t}}{\gamma_{t}}=r \mathrm{~d} t, \frac{\mathrm{d} S_{t}}{S_{t}}=r \mathrm{~d} t+\mathrm{d} B_{t}
$$

Replacing last equalities in (18), we obtain

$$
\begin{aligned}
\mathrm{d} X_{t} & =X_{t}\left(1-\pi_{t}\right) r \mathrm{~d} t+X_{t} \pi_{t}\left[r \mathrm{~d} t+\mathrm{d} B_{t}\right]-\mathrm{d} C_{t} \\
& =r X_{t} \mathrm{~d} t+X_{t} \pi_{t} \mathrm{~d} B_{t}-\mathrm{d} C_{t} .
\end{aligned}
$$

Hereafter, we will refer to eq. (19) as the wealth equation. Such an equation can be expressed also in integral form, when we refer to the discounted wealth process $\gamma_{t}^{-1} X_{t}$. 
Recall that, given two Itô processes $Y_{t}^{1}$ and $Y_{t}^{2}$, the product rule ensures that

$$
\mathrm{d}\left(Y_{t}^{1} Y_{t}^{2}\right)=Y_{t}^{1} \mathrm{~d} Y_{t}^{2}+Y_{t}^{2} \mathrm{~d} Y_{t}^{1}+\mathrm{d} Y_{t}^{1} \cdot \mathrm{d} Y_{t}^{2} .
$$

In our case, $Y_{t}^{1}:=\gamma_{t}^{-1}$, so that $\mathrm{d} Y_{t}^{1}=-r \gamma_{t}^{-1} \mathrm{~d} t$, while $Y_{t}^{2}:=X_{t}$, whose differential is given by eq. (19). Therefore,

$$
\begin{aligned}
\mathrm{d}\left(\gamma_{t}^{-1} X_{t}\right) & =\gamma_{t}^{-1} \mathrm{~d} X_{t}+X_{t} \mathrm{~d} \gamma_{t}^{-1}+\mathrm{d} \gamma_{t}^{-1} \cdot \mathrm{d} X_{t} \\
& =\gamma_{t}^{-1}\left[r X_{t} \mathrm{~d} t+X_{t} \pi_{t} \mathrm{~d} B_{t}-\mathrm{d} C_{t}\right]-r \gamma_{t}^{-1} X_{t} \mathrm{~d} t \\
& -r \gamma_{t}^{-1} \mathrm{~d} t\left[r X_{t} \mathrm{~d} t+X_{t} \pi_{t} \mathrm{~d} B_{t}-\mathrm{d} C_{t}\right] \\
& =\gamma_{t}^{-1} X_{t} \pi_{t} \mathrm{~d} B_{t}-\gamma_{t}^{-1} \mathrm{~d} C_{t}-r^{2} \gamma_{t}^{-1} X_{t}(\mathrm{~d} t)^{2} \\
& -r \gamma_{t}^{-1} X_{t} \pi_{t}\left(\mathrm{~d} t \cdot \mathrm{d} B_{t}\right)+r \gamma_{t}^{-1}\left(\mathrm{~d} t \cdot \mathrm{d} C_{t}\right) \\
& =\gamma_{t}^{-1} X_{t} \pi_{t} \mathrm{~d} B_{t}-\gamma_{t}^{-1} \mathrm{~d} C_{t},
\end{aligned}
$$

where last equality holds by virtue of standard stochastic calculus. Then, by taking into account the initial conditions $\gamma_{0}=1$ and $X_{0}=y$, we get

$$
\gamma_{t}^{-1} X_{t}=y+\int_{0}^{t} \gamma_{u}^{-1} X_{u} \pi_{u} \mathrm{~d} B_{u}-\int_{0}^{t} \gamma_{u}^{-1} \mathrm{~d} C_{u} .
$$

Definition 20. A portfolio/consumption process pair $(\pi, C)$ satisfying conditions of Def. 18 and 19 is said to be admissible in $\mathcal{M}$ for the initial wealth $y$ if $\left(\pi_{t} X_{t}^{y, \pi, C}\right) \in M_{*}^{2}(0, T)$ and there exists a nonnegative random variable $L \in L_{*}^{2}(0, T)$ such that the wealth process $X_{t}^{y, \pi, C}$ satisfies

$$
X_{t}^{y, \pi, C} \geq-L, \quad t \leq T, \text { q.s. } .
$$

The class of all admissible portfolio/consumption process pairs is denoted by $\mathcal{A}(y)$.

Finally, let us define the class $\mathcal{S}_{s, t}$ of $\mathbb{F}$-stopping times $\tau: \Omega \rightarrow[s, t]$, with $0 \leq s<t \leq T$. In particular, we set $\mathcal{S}:=\mathcal{S}_{0, T}$.

The class of portfolio/consumption process pair $(\pi, C)$ such that the stopped process $X_{\cdot \wedge \tau}^{y, \pi, C}$ satisfies eq. (21) for any stopping time $\tau \in \mathcal{S}$, is called $\mathcal{A}(y, \tau)$.

\subsection{Interval of no-arbitrage prices}

Roughly speaking, an American contingent claim $H$ is a contract which obliges the seller to pay a certain amount $H_{\tau} \geq 0$ if the buyer of that claim decides to 
exercise it at a (stopping) time $\tau$. In such a framework, one could be interested in establishing how this amount should be, in the sense that it should neither be too expensive from the buyer's point of view, nor too cheap from the seller's side. In other words, the goal is to determine the fair price to pay to the seller for his obligation at time $t=0$, to deliver the amount $H_{\tau} \geq 0$ to the buyer at a stopping time $\tau$, chosen by the buyer himself.

Definition 21. An American contingent claim is an $\mathbb{F}$-adapted random variable $H_{t} \in L_{*}^{2}\left(\Omega_{T}\right)$ with continuous paths. We shall denote the price of the American contingent claim at time $t=0$ by $H_{0}$.

Definition 22. For any $\sigma \in[\underline{\sigma}, \bar{\sigma}]$, consider the probability measure $P^{\sigma} \in \mathscr{P}$ such that $P^{\sigma}=P_{0} \circ\left(B^{0, \sigma}\right)^{-1}$, where $P_{0}$ is the probability measure in the probability space $\left(\Omega, \mathcal{B}(\Omega), P_{0}\right)$ and $B^{0, \sigma}=\int_{0}^{\cdot} \sigma_{s} \mathrm{~d} B_{s}$, as defined in eq. (7).

We can define the function

$$
u_{t}^{\sigma}:=\sup _{\tau \in \mathcal{S}_{t, T}} \mathbb{E}^{P^{\sigma}}\left[\gamma_{\tau}^{-1} H_{\tau}\right], 0 \leq t \leq \tau \leq T,
$$

as the American contingent claim price at time $t$, when the underlying $S$ has volatility rate $\sigma$.

Recall that we are considering financial markets whose dynamic evolves according to stochastic volatility models. The resulting volatility uncertainty implies the incompleteness of the financial market, which implies, in turn, the existence of more than one fair price for general contingent claims, American contingent claim included.

For these reasons, we have to establish an interval of no-arbitrage prices for American contingent claims. In order to do this, we introduce the lower hedging class and the upper hedging class.

Definition 23. Given an American contingent claim $H$, the lower hedging class is defined as

$$
\mathcal{L}:=\left\{y \geq 0 \mid \exists \tau \in \mathcal{S},(\pi, C) \in \mathcal{A}(-y, \tau): X_{\tau}^{-y, \pi, C} \geq-H_{\tau} \text { q.s. }\right\} .
$$

In an similar way, the upper hedging class is defined as

$$
\mathcal{U}:=\left\{y \geq 0 \mid \exists(\pi, C) \in \mathcal{A}(y): X_{\tau}^{y, \pi, C} \geq H_{\tau} \text { q.s., } \forall \tau \in \mathcal{S}\right\} .
$$

The largest amount $y \geq 0$ that enables an agent to recover the debt he incurred at $t=0$ by purchasing the claim is called lower hedging price for the American 
contingent claim

$$
h_{\text {low }}:=\sup \{y: y \in \mathcal{L}\} .
$$

The smallest value of initial capital $y \geq 0$ that allows an agent to fulfill his obligation without risk and whenever the buyer should choose to ask for the payment, is called upper hedging price for the American contingent claim

$$
h_{u p}:=\inf \{y: y \in \mathcal{U}\} .
$$

Remark 24. As it has been pointed out in [8], there exists an asymmetry in the definition of the classes $\mathcal{L}$ and $\mathcal{U}$, since the no-arbitrage condition holds just for some stopping time $\tau \in \mathcal{S}$ in $\mathcal{L}$, while the same condition holds for any stopping time $\tau \in \mathcal{S}$ in $\mathcal{U}$.

It is interesting to note that the sets $\mathcal{U}$ and $\mathcal{L}$ are connected intervals, in fact, analogously to what can be proven in the constrained market case, see, e.g., [7, Thm. 4.3], we have

Proposition 25. $y \in \mathcal{L}$ and $0 \leq z \leq y$ implies $z \in \mathcal{L}$. Analogously, $y \in \mathcal{U}$ and $z \geq y$ implies $z \in \mathcal{U}$.

The terms lower and upper arise by the fact that they actually represent the endpoints of an interval in which the American contingent claim price ranges, as it is guaranteed by the following result

Theorem 26. For $\sigma \in[\underline{\sigma}, \bar{\sigma}]$, the American contingent claim price at time $t=0, u_{0}^{\sigma}$, is such that

$$
0 \leq H_{0} \leq h_{\text {low }} \leq u_{0}^{\sigma} \leq h_{\text {up }} \leq \infty
$$

Proof. If $\mathcal{U}=\emptyset$, then trivially $h_{u p}=\infty$ and $h_{u p} \geq u_{0}$. Hence, we can suppose $\mathcal{U} \neq \emptyset$. By definition of $\mathcal{U}$, we deduce that there exists a pair $(\pi, C) \in$ $\mathcal{A}(y)$ such that $X_{\tau}^{y, \pi, C} \geq H_{\tau}$ q.s., for all $\tau \in \mathcal{S}$.

Hence, we have, for $\tau \in \mathcal{S}, \mathbb{E}_{G}\left[\gamma_{\tau}^{-1} X_{\tau}^{y, \pi, C}\right] \geq \mathbb{E}_{G}\left[\gamma_{\tau}^{-1} H_{\tau}\right]$, thanks to monotonicity of the $G$-expectation.

On the other hand, Prop. (11) ensures that the $G$-expectation is an upper expectation with respect to a measure $P \in \mathscr{P}$, so that

$$
\mathbb{E}_{G}\left[\gamma_{\tau}^{-1} X_{\tau}^{y, \pi, C}\right] \geq \sup _{P^{\sigma} \in \mathscr{P}} \mathbb{E}^{P^{\sigma}}\left[\gamma_{\tau}^{-1} H_{\tau}\right] \geq \mathbb{E}^{P^{\sigma}}\left[\gamma_{\tau}^{-1} H_{\tau}\right], \forall \tau \in \mathcal{S} .
$$


Hence, by definition of supremum over $\mathcal{S}$ and by eq. (22), we have

$$
\mathbb{E}_{G}\left[\gamma_{\tau}^{-1} X_{\tau}^{y, \pi, C}\right] \geq u_{0}^{\sigma} .
$$

At the same time, it results

$$
\begin{aligned}
\mathbb{E}_{G}\left[\gamma_{\tau}^{-1} X_{\tau}^{y, \pi, C}\right] & =\mathbb{E}_{G}\left[y+\int_{0}^{T \wedge \tau} \gamma_{t}^{-1} X_{t}^{y, \pi, C} \pi_{t} \mathrm{~d} B_{t}-\int_{0}^{T \wedge \tau} \gamma_{t}^{-1} \mathrm{~d} C_{t}\right] \\
& \leq \mathbb{E}_{G}\left[y+\int_{0}^{T \wedge \tau} \gamma_{t}^{-1} X_{t}^{y, \pi, C} \pi_{t} \mathrm{~d} B_{t}\right] \\
& \leq y+\mathbb{E}_{G}\left[\int_{0}^{T \wedge \tau} \gamma_{t}^{-1} X_{t}^{y, \pi, C} \pi_{t} \mathrm{~d} B_{t}\right] .
\end{aligned}
$$

thanks to eq. (20) and the monotonicity property of $G$-expectation.

Let us observe that the Itô integral depending on a stopping time satisfies eq. (16), in particular

$$
\mathbb{E}_{G}\left[\int_{0}^{T \wedge \tau} \gamma_{t}^{-1} X_{t}^{y, \pi, C} \pi_{t} \mathrm{~d} B_{t}\right]=\mathbb{E}_{G}\left[\int_{0}^{T} \mathbb{1}_{[0, \tau]}(t) \gamma_{t}^{-1} X_{t}^{y, \pi, C} \pi_{t} \mathrm{~d} B_{t}\right] \text { q.s. },
$$

where the integrand function $\mathbb{1}_{[0, \tau]}(t) \gamma_{t}^{-1} X_{t}^{y, \pi, C} \pi_{t}$ is in $M_{*}^{2}(0, T)$, for $X_{t}^{y, \pi, C} \pi_{t} \in$ $M_{*}^{2}(0, T)$ and Remark 17. Then we have

$$
\mathbb{E}_{G}\left[\int_{0}^{T \wedge \tau} \gamma_{t}^{-1} X_{t}^{y, \pi, C} \pi_{t} \mathrm{~d} B_{t}\right]=0
$$

by virtue of eq. (11), and we can use such an information in eq. (25) and then compare with eq. (24) to obtain $y \geq u_{0}^{\sigma}$, for all $y \in \mathcal{U}$. From the arbitrariness of $y$, we have $h_{u p} \geq u_{0}^{\sigma}$.

Analogously we can prove the remaining inequality. In fact, let us suppose that $y \in \mathcal{L} \neq \emptyset$, then, by definition, there exist $\tau \in \mathcal{S},(\pi, C) \in \mathcal{A}_{-}(-y, \tau)$ such that $X_{\tau}^{-y, \pi, C} \geq-H_{\tau}$ q.s. Therefore, proceeding as before we have

$$
\begin{aligned}
-y & \geq \mathbb{E}_{G}\left[-y+\int_{0}^{T \wedge \tau} \gamma_{t}^{-1} X_{t}^{-y, \pi, C} \pi_{t} \mathrm{~d} B_{t}\right] \\
& \geq \mathbb{E}_{G}\left[\gamma_{\tau}^{-1} X_{\tau}^{-y, \pi, C}\right] \geq \mathbb{E}_{G}\left[-\gamma_{\tau}^{-1} H_{\tau}\right] \\
& \geq-\sup _{\tau \in \mathcal{S}} \mathbb{E}^{P^{\sigma}}\left[\gamma_{\tau}^{-1} H_{\tau}\right]=-u_{0}^{\sigma},
\end{aligned}
$$

then $y \leq h_{u p}$, for all $y \in \mathcal{L}$ and the thesis follows thanks to the arbitrariness of $y$. 
The main goal of this Section is to achieve a free-arbitrage prices interval for American contingent claims . In order to do this, the definition of arbitrage opportunity has to be presented.

Definition 27. Let $u>0$ be the price of an American contingent claim $H$ in the market $\mathcal{M}$ at time $t=0$. There is an arbitrage opportunity in the financial market $(\mathcal{M}, u, H)$ if there exists either

(i) an admissible pair $(\pi, C) \in \mathcal{A}(y)$ such that

$$
\begin{aligned}
& X_{\tau}^{y, \pi, C} \geq H_{\tau} \text { q.s., } \forall \tau \in \mathcal{S}, \text { for some } 0<y<u, \\
& P\left(X_{\tau}^{y, \pi, C}-H_{\tau}>0\right)>0, \text { for at least one } P \in \mathscr{P},
\end{aligned}
$$

or

(ii) a stopping time $\tau \in \mathcal{S}$ and a pair $(\pi, C) \in \mathcal{A}(-y, \tau)$ such that

$$
\begin{aligned}
& X_{\tau}^{y, \pi, C}+H_{\tau} \geq 0 \text { q.s., for some } y>u \\
& P\left(X_{\tau}^{y, \pi, C}+H_{\tau}>0\right)>0, \text { for at least one } P \in \mathscr{P} .
\end{aligned}
$$

Let us give a couple of clarifications concerning the above Definition. First of all, the second condition in eqs. (26) and (27) is just the negation of the previous one. This allows to exclude that $X_{\tau}^{y, \pi, C} \pm H_{\tau}$ equals zero q.s.

Moreover we have a clear economical interpretation since, in the first case, an agent can sell the contingent claim at time $t=0$ for $u>y$, i.e., for more than is required to hedge it without risk throughout the interval $[0, T]$. While, in the second case, an agent can buy the contingent claim for $u<y$, that is, for less than the amount which allows him to recover his initial debt without risk, by exercising his option to the claim at some stopping time $\tau \in \mathcal{S}$. It follows that, in both cases, there exists an opportunity for creating wealth without risk. Clearly, any price $u>0$ that leads to such an arbitrage opportunity should be excluded and this is ensured by the following result.

Proposition 28. For any price $u_{0}$ such that $u_{0}>h_{u p}$ or $u_{0}<h_{\text {low }}$, there exists an arbitrage opportunity, while it does not happen for $u_{0}>0$ within the interval $\left[h_{\text {low }}, h_{\text {up }}\right]$.

Proof. Lemma 25 ensures that $\mathcal{U}$ and $\mathcal{L}$ are intervals. Therefore, suppose there exists a price $u>0$ of an American contingent claim such that $u>h_{u p}(K)$ and let $y \in\left(h_{u p}(K), u\right)$. This is equivalent to impose that $h_{u p}(K)<y<u$. 
The first inequality guarantees that $y \in \mathcal{U}$, so that, by definition, there exists a pair $(\pi, C) \in \mathcal{A}_{+}(y)$ such that $X_{\tau}^{y, \pi, C} \geq H_{\tau}$ q.s., for all $\tau \in \mathcal{S}$. This means that there exists a measure $P \in \mathscr{P}$ with $P\left(X_{\tau}^{y, \pi, C} \geq H_{\tau}\right)=1$.

On the other hand, since $y<u$, then there exists $a>1$ such that $a y=u \in$ $\mathcal{U}$ and so $X_{\tau}^{a y, \pi, a C}=a X_{\tau}^{y, \pi, C}$. Hence,

$$
1=P\left(X_{\tau}^{y, \pi, C} \geq H_{\tau}\right) \leq P\left(X_{\tau}^{a y, \pi, a C}>H_{\tau}\right)+P\left(X_{\tau}^{y, \pi, C}=H_{\tau}\right)
$$

or, equivalently,

$$
P\left(X_{\tau}^{a y, \pi, a C}>H_{\tau}\right) \geq 1-P\left(X_{\tau}^{y, \pi, C}=H_{\tau}\right)>0,
$$

which says that there exists an arbitrage opportunity.

The interval $\left[h_{\text {low }}(K), h_{u p}(K)\right]$ is called arbitrage-free price interval.

\section{Conclusion}

In this work we retrieve an inequality for American contingent claim prices at time $t=0$, involving the supremum, $h_{\text {low }}$, and the infimum, $h_{\text {up }}$, of two different suitable sets, $\mathcal{U}$ and $\mathcal{L}$, respectively, when such derivatives are traded in a financial market with uncertain volatility and in the nonlinear expectation $G$-framework.

The next step, which belongs to our ongoing research, will be to find the explicit expression of both the upper and the lower bounds of the free-arbitrage price interval.

\section{References}

[1] P. Artzner, F. Delbaen, J.-M. Eber, D. Heath, Coherent measures of risk, Math. Finance 9 (1999), 203-228.

[2] Avellaneda, M., Levy, A., Paras, A., (1995) Pricing and hedging derivative securities in markets with uncertain volatilities. Appl. Math. Finance 2, $73-88$.

[3] L. Denis, M. Hu, S. Peng, Function spaces and capacity related to a sublinear expectation: application to G-Brownian motion paths, Potential Anal. 34 (2010), 139-161. 
[4] N. El Karoui, M.C. Quenez, Dynamic Programming and Pricing of Contingent Claims in Incomplete Market, SIAM J. of Control and Optimization 33, No. 1 (1995).

[5] N. El Karoui, S. Peng, M.C. Quenez, Backward stochastic differential equations in finance, Math. Finance 7, No. 1 (1997), 1-71.

[6] Y. Hu, S. Peng, Some estimates for martingale representation under Gexpectation, arXiv:1004.1098v1, (2010).

[7] I. Karatzas, S.G. Kou, Hedging American contingent claims with constrained portfolios, Finance Stochast. 2, (1998), 215-258.

[8] I. Karatzas, S.G. Kou, On the pricing of contingent claims under constraints, Ann. Appl. Probab. 6, No. 2 (1996), 321-369.

[9] X. Li, S. Peng, Stopping times and related Itô's calculus with G-Brownian Motion, Atoc. Proc. Applications 121 (2011), 1492-1508.

[10] S. Peng, BSDE and related g-expectation, in Pitman Research Notes in Mathematics Series 364, Backward Stochastic Differential Equation, Ed. by N. El Karoui \& L. Mazliak (1997), 141-159.

[11] S. Peng, Filtration Consistent Nonlinear Expectations and Evaluations of Contingent Claims, Acta Math. Appl. Sin. 20, No. 2 (2004), 191-214.

[12] S. Peng, Nonlinear Expectations, Nonlinear Evaluations and Risk Measures. Stochastic Methods in Finance. In: Frittelli, M., Runggaldier, W. (Eds.), Lectures Notes in Mathematics, Springer (2004), 165-254.

[13] S. Peng, Nonlinear Expectations and Nonlinear Markov Chains, Chin. Annal Math. 26, No. 2 (2005), 159-184.

[14] S. Peng, G-Brownian motion and dynamic risk measure under volatility uncertainty. arXiv:0711.2834v1, (2007).

[15] E. Rosazza Gianin, Some examples of risk measures via g-expectations working paper 41, Dept. of Statistics and Quantitative Methods, University of Milano-Bicocca, (2002).

[16] H.M. Soner, N. Touzi, J. Zhiang, Martingale representation theorem for the G-expectation, Stoch. Proc. Appl. 121, No. 2 (2011), 265-287. 
[17] Y. Song, Some properties on G-evaluation and its applications to $G$ martingale decomposition, Sci. China Math. 54, No.2 (2011), 287-300.

[18] J. Vorbrink, Financial markets with volatility uncertainty, J. Math. Econom. 53, (2014), 64-78. 
